\title{
Platelet Rich Plasma as a Modality of Treatment for Primary Knee Osteoarthritis
}

\author{
R. S. Ayyappa Naidu¹, Mogulesh², C. N. S. Mounika³, D. Venkateswara Rao ${ }^{4}$, C. Shyam Kumar ${ }^{5}$, A. Srinivasa Rao ${ }^{6}$
}

${ }^{1}$ Tutor, Department of Orthopaedics, Siddhartha Medical College, Vijayawada, Andhra Pradesh, India. ${ }^{2}$ Junior Resident, Department of Orthopaedics, Siddhartha Medical College, Vijayawada, Andhra Pradesh, India. ${ }^{3}$ Assistant Professor, Department of Orthopaedics, Siddhartha Medical College, Vijayawada, Andhra Pradesh, India. ${ }^{4}$ Professor and HOD, Department of Orthopaedics, Siddhartha Medical College, Vijayawada, Andhra Pradesh, India. ${ }^{5}$ Professor, Department of Orthopaedics, Siddhartha Medical College, Vijayawada, Andhra Pradesh, India. ${ }^{6}$ Associate Professor, Department of Orthopaedics, Siddhartha Medical College, Vijayawada, Andhra Pradesh, India.

\section{ABSTRACT}

\section{BACKGROUND}

Osteoarthritis is a degenerative, chronic disease that affects up to $80 \%$ of the population over 75 years of age. ${ }^{1} \mathrm{~A}$ recent report indicates that knee osteoarthritis is likely to become the fourth most crucial aetiology of physical limitation in women and the eighth most in men. ${ }^{2}$ In middle-aged persons, osteoarthritis may cause a major burden in lost time at work and early retirement. The symptoms are often associated with notable functional impairment, as well as signs and symptoms of inflammation, including pain, stiffness, and loss of movement. Analgesics, nonsteroidal and steroidal anti-inflammatory drugs, glucosamine/chondroitin supplementation, physical therapy, and hyaluronic acid injections are useful in the treatment of osteoarthritis. New experimental studies have begun to target the biomechanical process of osteoarthritis with focus on repairing cartilage or replacement.

\section{METHODS}

In this study, 50 patients with knee joint osteoarthritis were treated with 3 monthly intraarticular knee joint injections of platelet-rich plasma under local anaesthesia as in and outpatient procedure in Government General Hospital, Siddhartha Medical College, Vijayawada, after taking clearance from ethical committee. All patients were treated with 3 monthly intra-articular injections of autologous platelet-rich plasma into the knee joint, under local anesthesia on an outpatient basis. All these cases were treated from January 2017 to August 2018.

\section{RESULTS}

From the data collected, results were analyzed and presented in this study. The average age incidence was 53 years with age distribution between 40-70 years. 32 patients $(64 \%)$ were overweight, $12(24 \%)$ patients were obese, and 6 patients $(12 \%)$ were normal weight. $24(48 \%)$ patients had grade 1,26 patients $(52 \%)$ had grade 2 osteoarthritis according to Kellgren-Lawrence grading system. VAS scale improved significantly from an average of 5.94 to 3.42. IKDC score increased significantly from 38.56 to 76.12 . Six patients experienced pain at the injection site, one patient had increasing pain, and one patient had skin discoloration at the injection site. No infections or allergies were noted.

\section{CONCLUSIONS}

PRP procedure showed a higher degree of efficacy as well as significant findings of more and longer pain reduction, improved function, and patient satisfaction. This was particularly noticeable in the treatment of younger patients with less severe articular cartilage degeneration. It could be utilized as a reasonable treatment option when other therapies fail or are inappropriate for the particular patient. However, more number of observations should be done to come to a conclusion.

\section{KEY WORDS}

Osteoarthritis, Degenerative Chronic Disease, Platelet-Rich Plasma, KellgrenLawrence Scoring, VAS Scale, IKDC Score

\author{
Corresponding Author: \\ Dr. C. N. S. Mounika, \\ Assistant Professor, \\ Department of Orthopaedics, \\ Siddhartha Medical College, \\ Vijayawada, Andhra Pradesh, India. \\ E-mail: cnsmounika@gmail.com
}

DOI: 10.14260/jemds/2019/665

Financial or Other Competing Interests: None.

How to Cite This Article:

Naidu RSA, Mogulesh, Mounika CNS, et al. Platelet rich plasma as a modality of treatment for primary knee osteoarthritis. J. Evolution Med. Dent. Sci. 2019;8(41): 3057-3063, DOI:

$10.14260 /$ jemds/2019/665

Submission 25-02-2019,

Peer Review 24-09-2019,

Acceptance 01-10-2019,

Published 14-10-2019. 


\section{BACKGROUND}

Osteoarthritis is a degenerative, chronic disease that affects up to $80 \%$ of the population over 75 years of age. ${ }^{1} \mathrm{~A}$ recent report indicates that knee osteoarthritis is likely to become the fourth most crucial aetiology of physical limitation in women and the eighth most in men. ${ }^{2}$ In middle-aged persons, osteoarthritis may cause a major burden in lost time at work and early retirement. Non-modifiable risk factors for osteoarthritis are gender and age whereas the modifiable risk factors are body mass index, physical activity ${ }^{3}$, etc. Recent prospective studies have established that obesity is a primary risk factor for knee osteoarthritis. Increased loading of the knee joints can lead to cartilage breakdown and failure of components of structural support. ${ }^{4}$ Before 45 years of age, the prevalence of osteoarthritis in most joints is higher in men than in women. After about age 45 years, women are more often affected than men. ${ }^{5}$ In some studies, women have shown increased rates of cartilage loss and progression of knee cartilage defects than men. ${ }^{6}$ Analgesics, non-steroidal and steroidal anti-inflammatory drugs, glucosamine/ chondroitin supplementation, physical therapy, and hyaluronic acid injections are useful in the treatment of osteoarthritis. However, most of them not addressing the pathology or have shown only minor benefits and significant side effects.7,8,9 New experimental studies have begun to target the biomechanical process of osteoarthritis with the focus on repairing cartilage or replacement. Autologous platelet-rich plasma therapy has got particular attention, in which high concentration of platelets achieved in a short volume of plasma after being placed in a centrifuge. Platelets play a vital part in tissue homeostasis.

Our chief aim was to study the effects of intra-articular injections of Platelet-rich plasma in the management of knee joint osteoarthritis and to compare the results with standard studies and draw conclusion.

\section{METHODS}

This is a prospective observational study, conducted among 50 patients (selected at conveniences) with knee osteoarthritis who were studied in the outpatient department of Orthopaedics, Government General Hospital, attached to Siddhartha Medical College, Vijayawada. The hospital ethics committee approved the study. An informed written consent was taken from all the participants. Patients were diagnosed using. The American College of Rheumatology (ACR) classification criteria of osteoarthritis. All patients were treated with 3 monthly intra-articular injections of autologous platelet-rich plasma into the knee joint, under local anesthesia on an outpatient basis. All these cases were treated from January 2017 to August 2018. A detailed history was taken. A preliminary general and physical examination were done. Build and nourishment noted. Systemic examination of the cardiovascular, respiratory, gastrointestinal and genitourinary examination was followed as routine. A detailed local examination of the knee joint was done. Data was entered in MS Excel 2007 and analysed.
Preparation of autologous platelet-rich plasma (PRP)- $30 \mathrm{ml}$ of venous blood was taken from every patient and collected in sterile sodium citrated tubes. The tubes with citrated blood were centrifuged at $1800 \mathrm{rpm}$ for $15 \mathrm{~min}$ to separate erythrocytes, and at $3500 \mathrm{rpm}$ for $10 \mathrm{~min}$ to concentrate platelets. By this method, 3 - $5 \mathrm{ml}$ of platelet-rich plasma were obtained and injected immediately without storage. It has been stated that using freshly harvested PRP might preserve all the platelet functions better.

PRP knee intra-articular injection technique- The injection was given in the supine position and with all aseptic precautions. The affected side was exposed up to the thigh and cleaned with betadine scrub (7.5\%) and spirit. Then painted with $5 \%$ betadine solution and draped with linen towels. The knee joint was palpated, and good understanding of anatomical configuration was made. 2\% Xylocaine injection was given in the skin and soft tissues of the lateral aspect of knee joint. The leg was held firm in neutral rotation, and intra-articular injection of autologous platelet-rich plasma was given by the lateral approach with knee in complete extension using a 23-gauge needle. After injection, patients were instructed not to use the injected leg for $24 \mathrm{~h}$, use ice packs over the injected joint and not to use NSAIDs during this period.

\section{Inclusion Criteria}

Patients with history of chronic pain (of at least 4 months duration) or swelling of the knee, not responding to NSAIDs and/or physical therapy with radiographic findings of grade 1 (definite osteophyte, unimpaired joint space) and grade 2 (moderate diminution of joint space osteoarthritis of the knee joint) according to Kellgren- Lawrence scale were included in the study.

\section{Exclusion Criteria}

Patients with diabetes, rheumatoid arthritis, major axial deviation (varus of more than $5 \mathrm{deg} /$ valgus more than 5 deg), haematological diseases (coagulopathies), severe cardiovascular diseases, infections, immunosuppression, patients on therapy with anticoagulants-anti aggregants or nonsteroidal anti-inflammatory drugs within 5 days before blood donation, were excluded from the study.

\section{Follow up Assessment}

After 6 months, all patients were re-evaluated by physical examination, assessment of visual analog scale for pain, international knee documentation committee (IKDC) score.

\section{Statistical Analysis}

Chi-square test was used to assess differences between quantitative and qualitative data at baseline and after 3 PRP injections. Spearman's correlation coefficient analysis was performed to identify factors associated with better functional outcomes. The clinical features were evaluated using the chi-square test. The VAS scale and IKDC score were assessed using chi square test. A statistically significant cutoff value was set at $\mathrm{p}<0.05$. 
RESULTS

After 6 months, all patients were re-evaluated according to Age, Sex, BMI (Body mass index), Side involved, Severity (Grade) of osteoarthritis, Pre and post-PRP injection comparison of crepitus, local temperature, Joint line tenderness, Effusion, Range of motion, Visual Analog Scale 12 for pain, International Knee Documentation Committee (IKDC) score(Table 2). The maximum and minimum age in this study was found to be 41 years and 70 years. The average age of the patients was calculated as the total age of patients / no. Of patients $=52.68$ yrs. Out of 50 patients, 32 were females, and 28 were males Out of 50 knee joints treated, 28 were the right side and 22 were the left side. 32 patients were found to be overweight, 12 were obese, and 6 were normal weight. The grading of osteoarthritis of the knee.

\section{Kellgren and Lawrence Classification:10,11}

Grade 1 (Doubtful):

Minute osteophyte, doubtful significance.

Grade 2 (Mild):

Definite osteophyte, unimpaired joint space.

Grade 3 (Moderate):

Moderate diminution of joint space.

Grade 4 (Severe):

Joint space greatly impaired with sclerosis of subchondral bone.

Out of 50 study patients, 24 patients had grade 1 osteoarthritis, and 26 patients had grade 2 osteoarthritis. The clinical features were evaluated using the chi-square test. The VAS ${ }^{12}$ scale and IKDC ${ }^{13}$ score were assessed using the chi square test.

\begin{tabular}{|c|c|c|}
\hline Kellgren-Lawrence Scale $\mathbf{1 0}^{\mathbf{1 1 1}}$ & No. of Patients & Percentage \\
\hline Grade 1 (Doubtful) & 24 & $48 \%$ \\
\hline Grade 2 (Mild) & 26 & $52 \%$ \\
\hline Grade 3 (Moderate) & 0 & $0 \%$ \\
\hline Grade 4 (Severe) & 0 & $0 \%$ \\
\hline Table 1. Kellgren-Lawrence Grading of Patients with Primary \\
Osteoarthritis \\
\hline
\end{tabular}

\begin{tabular}{|c|c|c|c|c|}
\hline Clinical Data & $\begin{array}{c}\text { Baseline } \\
\text { (No. } \\
\text { Patients } \\
\text { out of 50) }\end{array}$ & $\begin{array}{c}\text { After 6 Months } \\
\text { Follow Up } \\
\text { (No. of Patients } \\
\text { out of 50) }\end{array}$ & $\begin{array}{c}\text { Chi- } \\
\text { Square } \\
\text { Value }\end{array}$ & $\begin{array}{c}\mathbf{p} \\
\text { Value }\end{array}$ \\
\hline Local rise of temp & 5 & 0 & 7.76 & $<0.005$ \\
\hline Tender joint line & 33 & 15 & 12.98 & $<0.001$ \\
\hline Crepitus & 33 & 20 & 6.78 & $<0.01$ \\
\hline Effusion & 5 & 5 & 0 & 1 \\
\hline Limited range of movements & 18 & 5 & 9.54 & $<0.002$ \\
\hline Table 2. Clinical Evaluation of Patients with Primary Osteoarthritis \\
after PRP Injection \\
\hline
\end{tabular}

\section{DISCUSSION}

Osteoarthritis is a major public health problem which causes pain and disability in one-third of all affected patients. The symptoms are often associated with notable functional impairment, as well as signs and symptoms of inflammation, including pain, stiffness, and loss of movement. ${ }^{14}$ Multiple factors are known to affect the progression of $\mathrm{OA}$, including joint instability and/or malalignment, obesity, increasing age, associated intra-articular crystal deposition, muscle weakness, and peripheral neuropathy. Advances in molecular biology raise hopes that new therapeutic targets will be identified that will allow more than just symptomatic therapy. Joint replacement is still the unsurpassed therapy for advanced and incapacitating OA.

However, with increasing appreciation of the contribution of all three joint compartments to disease progression, research in OA pathogenesis, biomarkers, and treatment has broadened immensely, and many new potential therapeutic targets have emerged over the past years. The growing interests in the use of PRP in OA treatment, which might provide cellular and humoral mediators to promote tissue healing and repair have gained momentum in the past few years and led to several studies. ${ }^{15}$ Autologous plasma is a biological therapy approach with the goal of delivering concentrated platelets to accelerate and support the healing of injuries to hard and soft tissue without exposing the patient to major risks. ${ }^{16}$ Growth factors, an essential part of PRP, induce differentiation of mesenchymal stem cells into chondrocytes and thereby increase cell proliferation. They also suppress inflammatory mediators such as interleukin-1, encourage matrix deposition, and slow down catabolism. ${ }^{17}$ Hence, growth factors help stabilize cartilage homeostasis and possibly reverse articular degeneration. The use of autologous blood versus synthetic chemicals also eliminates the risk for allergic reaction or disease transfer in addition to limiting possible drug toxicity. ${ }^{18}$ The simple and efficient in-house preparation at the time of patient visit proves to be of advantage for the PRP procedure.

In our study 50 patients with Grade 1 or Grade 2 knee osteoarthritis treated with 3 monthly injections of plateletrich plasma and following conclusion made after six months of follow up. The average age documented was 52.68 years. Osteoarthritis of the knee was common in $5^{\text {th }}$ and $6^{\text {th }}$ decade of life, the commonest in between 51- and 55-years age group. In this study, 41 patients were found to be below the age of 60 years. Also, results were found to be better in the younger patients when compared to the older. In this study 32 patients (64\%) were females, and 18 (36\%) patients were male. The baseline visual analog score ${ }^{12}$ in female patients was 5.93 and 3.45 at 6 months, and that in males was 5.98 (Baseline) and 3.36 (at 6 Months) thus showing that there is no notable difference in response to treatment between males and females. The value of IKDC ${ }^{13}$ score in males was 38.75 (Baseline) and 76.08 (at 6 Months) and that in females was 38.42 (Baseline) and 76.14 (at 6 Months) therefore showing no significant difference in response to treatment in between males and females.

Anderson JJ, Felson $\mathrm{DT}^{3}$ in his study found that overweight is the most potent risk factor for knee osteoarthritis. In our study 32 patients (64\%) were overweight (BMI 25-29.9) and 6 (12\%) were normal weight (BMI 18.5-24.9) and 12 (24\%) were obese (BMI >30). Adipose tissue, once thought a passive storage portal of energy, is now recognized as an extremely metabolic endocrine organ with the ability to secrete adipocytokines, such as leptin, adiponectin, and resistin. BMI and body weight are consistently correlated with leptin levels among those with knee osteoarthritis. Leptin and its receptors have 
been identified in human chondrocytes, osteophytes, synovium, and infrapatellar fat pad and may affect growth factor synthesis and anabolism.19,20,21 Examination of cartilage, subchondral bone, and osteophytes shows evidence of upregulated leptin expression. Leptin expression has been directly associated with the degree of cartilage degeneration and synergistic relationships of leptin and proinflammatory cytokines have been reported.19,22 Following administration of leptin, chondrocytes from osteoarthritic patients, had increased production of Interleukin-1b, Matrix metalloproteinase 9 and 13. This suggests that leptin has a direct pro-inflammatory and catabolic role in cartilage metabolism and leptin or its receptor may serve as a therapeutic target. ${ }^{22}$

Higher concentrations of adiponectin within the joint suggest an essential role for adiponectin in osteoarthritis development. Adiponectin expression was 100 -fold higher in the synovial fluid of osteoarthritis patients as compared with their plasma.22 Furthermore, the adiponectin receptor AdipoR1 was expressed in cartilage, bone, and synovial tissues. Demonstration of down regulation of Matrix metalloproteinase-13 and up regulation of an associated inhibitor suggests that adiponectin may be protective in the progression of osteoarthritis. ${ }^{23}$

In addition to the adipocytokines, obesity may cause other systemic effects related to osteoarthritis, including roles for disordered glucose and lipid metabolism. Metabolic changes resultant from insulin resistance and increased glucose load are closely related to proinflammatory cytokine production, characteristic of a chronic inflammatory state. Furthermore, the formation of advanced glycation end products (AGEs) may be associated with increased collagen stiffness, alterations in the mechanical properties of the extracellular matrix, and decreased proteoglycan synthesis, thereby possibly resulting in cartilage degradation. ${ }^{24}$

In this study patients with Grade 1 and Grade 2 osteoarthritis were selected according to KellegrenLawrence scale ${ }^{10,11}$ which included 24 (48\%) grade 1, 26 (52\%) grade 2 osteoarthritis cases and Grade 1 osteoarthritis patients has better improvement of symptoms than grade 2 patients. In the study conducted by Elizaveta Kon and R Buda, 25 there were $30 \%$ cases of minimal and 70 percent cases of moderate osteoarthritis knee. According to the study of Elizaveta Kon et al. ${ }^{25}$ which examined PRP effectiveness on the knee joint, better results were achieved in patients with a low degree of cartilage degeneration.

Sampson S et al. ${ }^{26}$ noted in a study of 14 patients' cartilage thicknesses measured by ultrasonography, that responses to PRP decreased as the grade of osteoarthritis and chondropenia increased. As joint degeneration progress, factors such as decrease of viable cells, muscle function loss, joint instability due to increased ligament laxity, reduction in anabolic response to growth factors, loss of chondrocyte and thinning of cartilage plate may reduce the effectiveness of PRP.

Despite poorer results, patients with advanced osteoarthritis still benefit from PRP. In a comparative study of PRP and HA in grade 1-3 knee osteoarthritis, the PRP group showed significantly better outcomes after 6 months, and the worst results were observed in subjects treated with HA having grade 3 knee OA.27 Elizaveta Kon et al. ${ }^{25}$ hypothesize that additional biological mechanisms, not currently known, are responsible for the improvement of $\mathrm{OA}$ symptoms after PRP treatment. In the advanced stages of osteoarthritis, PRP might not have a direct impact on the chondrocyte metabolic process, but an anti-inflammatory effect through the control of joint homeostasis and the cytokine level.25,28

\section{Pre and Post-Intra-Articular PRP Injection Status}

In our study, significant improvement noted in Joint line tenderness $(p<0.001)$, Local rise of temperature $(p<0.005)$, Crepitus $(p<0.01)$, Restriction of movements $(p<0.002)$. The joint effusion did not show any improvement $(p=1)$. The joint line tenderness is the parameter which was found to improve most significantly. Ali Soliman Hassan a, Abeer Mohamed El-Shafey a, Hanan S. Ahmed ${ }^{29}$ in their study statistically significant improvement was observed regarding most of the clinical aspects, such as tenderness over the joint line, crepitus, and restriction of movements. There was, also, improvement in the number of patients having hotness, effusion and Baker's cyst, but this improvement did not reach a statistically notable level.

\section{VAS ${ }^{12}$ Score and IKDC ${ }^{13}$ Score}

The baseline VAS 12 scale score (Maximum out of 10) was $5.94+/-0.79$ (SD) has shown improvement to $3.4+/-0.68$ (SD) which is significant with chi square-test to $<0.001$ (highly significant). The IKDC ${ }^{13}$ score (Maximum 100) baseline was 38.56 and that at 6 months follow up was 76.12 which when compared with chi square test was having a pvalue of $<0.001$ (Highly significant). Elizaveta Kon et al. ${ }^{25}$ conducted a prospective study in 2010 in which 100 patients with knee osteoarthritis were treated with Platelet-rich plasma and assessed at six and twelve months through $\mathrm{IKDC}^{13}$ and EQ-VAS ${ }^{30}$ scales. There was a positive response in the first 6 months, not sustained after 12 months, despite remaining significantly above the original scores. Another study by the same group, with comparable methods, published in 2010 by Filardo et al. ${ }^{17}$ Demonstrated a positive response in the first twelve months, which was not sustained until the end of the second year of follow-up. Kon E et al. ${ }^{30}$ In 2011 treated three groups of 50 patients with knee OA who received PRP, low and high molecular weight HA respectively. Random allocation of patients was not carried out among the three groups, since the treatment performed was dependent on the institution where the injections were performed, with each institution being accountable for the application of a single substance. Clinical response evaluated by the IKDC ${ }^{13}$ and EQ-VAS ${ }^{30}$ scores were higher in patients group that received platelet-rich plasma injections compared to hyaluronic acid. The low molecular weight hyaluronic acid performed better than the high molecular weight; however, still lower than the response obtained by PRP.

In 2012, Jang et al. ${ }^{31}$ Studied the effect of a single application of PRP in 65 patients with knee osteoarthritis. The patient showed a favourable response in the visual analog scale 12 for pain, and IKDC ${ }^{13}$ scores up to 6 months after treatment, which was not maintained after one year of the injection. In 2012, Filardo et al. ${ }^{32}$ Also compared PRP with HA in 109 patients with OA of the knee through a randomized, double-blind control study. The patients were assessed by IKDC13, EQ-VAS ${ }^{30}$ (Visual analog scale Euro Qol), Tegner, 33 and KOOS $^{34}$ scores throughout 12 months. Both 
groups showed clinical improvement, with no distinction between them. When comparing the groups concerning the degree of osteoarthritis in the scale of Kellgren-Lawrence ${ }^{10,11}$ only a tendency toward better response from PRP in milder degree (Degrees $\leq$ II) was found.

In 2013, Hart R et al. ${ }^{35}$ Performed a sequence of nine PRP applications after undergoing arthroscopy in 50 patients with knee osteoarthritis. The assessment tools were Tegner, ${ }^{33}$ Lyshcolm, ${ }^{30}$ Cincinnati ${ }^{36}$ and IKDC ${ }^{13}$ scores. There was the betterment of the indices after 6 months of treatment, which was not maintained after 12 months. Another study published in 2013 led by Say F et al. ${ }^{37}$ Compared a single PRP injection with three hyaluronic acid injections in individuals with knee osteoarthritis.

Clinical evaluation was made by VAS (Visual Analog Scale), ${ }^{12}$ and KOOS (Knee injury and Osteoarthritis Outcome Score $)^{34}$ scores. The study showed clinical improvement with both treatments after 3 and 6 months of the procedures, however, with better improvement in patients treated with PRP. Besides, the cost of treatment was lower in the group managed with PRP. The limitations stated by the author were no patient's randomization and the exclusive use of clinical parameters in the results analysis.

Sánchez et al..$^{38}$ In 2012, in a double-blinded, randomized control trial, compared the PRP and HA in 176 patients with knee OA. The scores used for the interpretation were WOMAC $^{39}$ (Western Ontario McMasters Universities Osteoarthritis Index) and Lequesne. ${ }^{40}$ Treatment with PRP reduced $50 \%$ of the WOMAC 39 index (Primary outcome) and noted a trend towards betterment in secondary outcomes, however with no statistical significance. The limitations stated by the author were the short follow-up, no comparison between the level of physical activity before and after treatment, the lack of a placebo group and the exclusion of cases considered to be severe on radiological examination.

Vaquerizo et al. ${ }^{41}$ Published in 2013 a study with a similar design to the study by Sanchez, where 96 patients were assessed for 48 weeks. The PRP showed better outcome in all parameters assessed, both in 24 and in 48 weeks, including the percentage of responders of Outcome Measures for Rheumatology Committee and Osteoarthritis Research Society International Standing Committee for Clinical Trials Response Criteria Initiative. ${ }^{42}$

The overall results were promising without any major procedural complications. This was especially noticeable in the younger population and with less severe osteoarthritis. Older and more degenerative joints tend to have less viable cells, and with that, a smaller potential for growth factor response might be the reason behind the low improvements in patients over the age of 50 and with severe osteoarthritis. Despite lower results, patients with advanced OA still benefited from Platelet-rich plasma

\section{Differences in the Method of Preparation and Administration of PRP}

In this study PRP was prepared by using $30 \mathrm{ml}$ of venous blood to which anticoagulant $3.2 \%$ buffered Tri-sodium citrate was added and centrifuged at $1800 \mathrm{rpm}$ for 15 minutes to separate RBC and then at $3500 \mathrm{rpm}$ for $10 \mathrm{~min}$ to obtain $3-5 \mathrm{ml}$ of PRP to which calcium chloride was added for activation of platelets to release growth factors.
Each study prepared their PRP somewhat differently. Ali Soliman, Abeer ${ }^{29}$ used centrifugation at the same speed of $1800 \mathrm{rpm}$ for $18 \mathrm{~min}$ and then $3500 \mathrm{rpm}$ for $10 \mathrm{~min}$. Elizaveta Kon et al. ${ }^{25}$ Separated the blood sample twice at $1480 \mathrm{rpm} \times 6$ minutes and again at $3400 \mathrm{rpm} \times 15$ minutes. Though not explicitly stated, it appears that M. Sanchez et al. ${ }^{38}$ Utilized an enzyme-linked immunosorbent assay kit to quantify the number of platelets and growth factors. Also, Elizaveta Kon et al. ${ }^{25}$ Added calcium chloride before administration to activate the platelets; an anticoagulation agent was not used. M. Sanchez et al. ${ }^{38}$ opted, not only to add an anticoagulation agent but also activate the platelets via calcium chloride. In our study about $3-5 \mathrm{ml}$ of PRP was injected and in other studies administered a different dose to their test subject. Elizaveta Kon et al. ${ }^{25}$ injected $5 \mathrm{ml}$ of PRP, whereas M. Sanchez et al. ${ }^{38}$ used 6 to $8 \mathrm{ml}$ of PRP in their injections. Therefore, the method of preparation of PRP, amount of PRP injected and the concentration of platelets achieved in platelet-rich plasma may contribute to the differences in response to the level of improvement achieved

\section{Effects of Over or Underestimation of Answering the VAS $^{12}$ Scale and IKDC ${ }^{13}$ Score by the Patients}

There is also the possibility of over or underestimation by the patient when answering the IKDC,13 VAS,12 or WOMAC ${ }^{39}$ questionnaires which are subjective and hence resulting in recall bias can influence the overall results.

\section{Complications}

Side effects related to the injection of platelet-rich plasma are considered uncommon and, when present, usually manifest in a mild and self-limited form. In our study, 6 patients experienced slight pain at the site of injection which lasted for one week and only one patient experienced marked pain. One patient had skin discoloration in the form of bruising. No cases suffered from infection or allergic reaction. Local symptoms are the most usual adverse events, ranging from pain at the injection site to signs of arthritis. Filardo et al. ${ }^{17}$ in 2011 showed that the way of attainment of PRP determines the degree of intra-articular inflammatory response, with this effect being attributed to the no. of leukocytes present in the infiltrate. Allergic reactions are possible but rare since it is an autologous product. The most dreaded complication is the intra-articular infection that can be prevented by performing the aseptic procedure. In other studies, the most frequently reported adverse events were arthralgia in the injected joint, whose severity varied from mild to moderate, and its resolution occurred in days, extending to weeks in the most severe cases. M. Sánchez et al. ${ }^{38}$ Reported a case of rash after the injection, which resolved spontaneously, with no need for specific treatment. Filardo et al. ${ }^{32}$ Showed that higher postinjection pain was noted in those patients injected with PRP compared to HA. Systemic symptoms and infections were not reported in the analysed studies.

\section{CONCLUSIONS}

PRP procedure showed a higher degree of efficacy as well as significant findings of more and longer pain reduction, improved function, and patient satisfaction. This was particularly noticeable in the treatment of younger patients 
with less severe articular cartilage degeneration. All of the comparative studies suggest that PRP injections are a useful approach and an alternative in the treatment of OA. This minimally invasive procedure appears to be safe and effective. It could be utilized as a reasonable treatment option when other therapies fail or are inappropriate for the particular patient.

\section{REFERENCES}

[1] Recht MP, Resnick D. Magnetic resonance imaging of articular cartilage: an overview. Top Magn Reson Imaging 1998;9(6):328-36.

[2] Lopez AD Murray CJL. The global burden of disease. World Health Organization 1997.

[3] Anderson JJ, Felson DT. Factors associated with osteoarthritis of the knee in the first national health and nutrition examination survey (HANES 1). Evidence for an association with overweight, race and physical demands for work. Am J Epidemiol 1988;128(1):179-89.

[4] Cooper C, Snow S, McAlindon TE, et al. Risk factors for the incidence and progression of radiographic knee osteoarthritis. Arthritis Rheum 2000;43(5):995-1000.

[5] Davis MA, Ettinger WH, Neuhaus JM, et al. Sex differences in osteoarthritis of the knee. The role of obesity. Am J Epidemiol 1988;127(5):1019-30.

[6] Hanna FS, Teichtahl AJ, Wluka AE, et al. Women have increased rates of cartilage loss and progression of cartilage defects at the knee than men: a gender study of adults without clinical knee osteoarthritis. Menopause 2009;16(4):666-70.

[7] Towheed TE, Maxwell L, Judd MG, et al. Acetaminophen for osteoarthritis. Cochrane Database Syst Rev 2006;(1):CD004257.

[8] Garner SE, Fidan DD, Frankish R, et al. Rofecoxib for osteoarthritis. Cochrane Database Syst Rev 2005;(1):CD005115.

[9] Wielage RC, Myers JA, Klein RW, et al. Cost-effectiveness analyses of osteoarthritis oral therapies: a systematic review. Appl Health Econ Health Policy 2013;11(6):593618.

[10] Kellgren JH, Lawrence JS. Radiological assessment of osteo-arthritis. Ann Rheum Dis 1957;16(4):494-502.

[11] Petersson IF, Boegard T, Saxne T, et al. Radiographic osteoarthritis of the knee classified by the Ahlbäck and Kellgren \& Lawrence systems for the tibiofemoral joint in people aged 35-54 years with chronic knee pain. Ann Rheum Dis 1997;56(8):493-6.

[12] Carlsson AM. Assessment of chronic pain. I. Aspects of the reliability and validity of the visual analogue scale. Pain 1983;16(1):87-101.

[13] Irrgang JJ, Anderson AF, Boland AL, et al. Development and validation of the international knee documentation committee subjective knee form. Am J Sports Med 2001;29(5):600-13.

[14] Felson DT. Clinical practice. Osteoarthritis of the knee. N Engl J Med 2006;354(8):841-8.

[15] Foster TE, Puskas BL, Mandelbaum BR, et al. Plateletrich plasma: from basic science to clinical applications. Am J Sports Med 2009;37(11):2259-72.
[16] Alsousou J, Thompson M, Hulley P, et al. The biology of platelet-rich plasma and its application in trauma and orthopedic surgery: a review of the literature. J Bone Joint Surg Br 2009;91(8):987-96.

[17] Filardo G, Kon E, Buda R, et al. Platelet-rich plasma intra-articular knee injections for the treatment of degenerative cartilage lesion and osteoarthritis. Knee Surg Sports Traumatol Arthrosc 2011;19(4):528-35.

[18] Sampson S, Gerhardt M, Mandelbaum B. Platelet-rich plasma injection grafts for musculoskeletal injuries: a review. Curr Rev Musculoskelet Med 2008;1(3-4):16574.

[19] Dumond H, Presle N, Terlain B, et al. Evidence for a key role of leptin in osteoarthritis. Arthritis Rheum 2003;48(11):3118-29.

[20] Presle N, Pottie P, Dumond H, et al. Differential distribution of adipokines between serum and synovial fluid in patients with osteoarthritis. Contribution of joint tissues to their articular production. Osteoarthritis Cartilage 2006;14(7):690-5.

[21] Gegout PP, Francin PJ, Mainard D, et al. Adipokines in osteoarthritis: friends or foes of cartilage homeostasis? Joint Bone Spine 2008;75(6):669-71.

[22] Simopoulou T, Malizos KN, Iliopoulos D, et al. Differential expression of leptin and leptin's receptor isoform (Ob-Rb) mRNA between advanced and minimally affected osteoarthritic cartilage; effect on cartilage metabolism. Osteoarthritis Cartilage 2007;15(8):872-83.

[23] Chen TH, Chen L, Hsieh MS, et al. Evidence for a protective role for adiponectin in osteoarthritis. Biochim Biophys Acta 2006;1762(8):711-8.

[24] DeGroot J. The AGE of the matrix: chemistry, consequences and cure. Curr Opin Pharmacol 2004;4(3):301-5.

[25] Kon E, Buda R, Filardo G, et al. Platelet-rich plasma: intra-articular knee injections produced favorable results on degenerative cartilage lesions. Knee Surg Sports Traumatol Arthrosc 2010;18(4):472-9.

[26] Sampson S, Reed M, Silvers H, et al. Injection of plateletrich plasma in patients with primary and secondary knee osteoarthritis: a pilot study. Am J Phys Med Rehabil 2010;89(12):961-9.

[27] Cerza F, Carni S, Carcangiu A, et al. Comparison between hyaluronic acid and platelet-rich plasma, intra-articular infiltration in the treatment of gonarthrosis. Am J Sports Med 2012;40(12):2822-7.

[28] Marmotti A, Rossi R, Castoldi F, et al. PRP and articular cartilage: a clinical update. Biomed Res Int 2015:2015:542502.

[29] Hassan AS, El-Shafey AM, Ahmed HS, et al. Effectiveness of the intra-articular injection of platelet-rich plasma in the treatment of patients with primary knee osteoarthritis. The Egyptian Rheumatologist 2015;37(3):119-24.

[30] Brooks R. EuroQol: the current state of play. Health Policy 1996;37(1):53-72.

[31] Kon E, Mandelbaum B, Buda R, et al. Platelet-rich plasma intra-articular injection versus hyaluronic acid viscosupplementation as treatments for cartilage 
pathology: from early degeneration to osteoarthritis. Arthroscopy 2011;27(11):1490-501.

[32] Filardo G, Kon E, Martino AD, et al. Platelet-rich plasma vs hyaluronic acid to treat knee degenerative pathology: study design and preliminary results of a randomized controlled trial. BMC Musculoskelet Disord 2012;13:229.

[33] Tegner Y, Lysholm J. Rating systems in the evaluation of knee ligament injuries. Clin Orthop Relat Res 1985;198:43-9.

[34] Roos EM, Roos HP, Lohmander LS, et al. Knee Injury and Osteoarthritis Outcome Score (KOOS)--development of a self-administered outcome measure. J Orthop Sports Phys Ther 1998;28(2):88-96.

[35] Hart R, Safi A, Komzak M, et al. Platelet-rich plasma in patients with tibiofemoral cartilage degeneration. Arch Orthop Trauma Surg 2013;133(9):1295-301.

[36] Noyes FR, Barber SD, Mooar LA. A rationale for assessing sports activity levels and limitations in knee disorders. Clin Orthop Relat Res 1989;(246):238-49.

[37] Say F, Gürler D, Yener K, et al. Platelet-rich plasma injection is more effective than hyaluronic acid in the treatment of knee osteoarthritis. Acta Chir Orthop Traumatol Cech 2013;80(4):278-83.
[38] Sanchez M, Anitua E, Azofra J, et al. Intra-articular injection of an autologous preparation rich in growth factors for the treatment of knee OA: a retrospective cohort study. Clinical Experimental Rheumatology 2008;26:910-3.

[39] Bellamy N. WOMAC Osteoarthritis Index user guide. London (Ontario, Canada): University of Western Ontario 1995.

[40] Lequesne MG, Mery C, Samson M, et al. Indexes of severity for osteoarthritis of the hip and knee. Validation--value in comparison with other assessment tests. Scand J Rheumatol Suppl 1987;65:85-9.

[41] Vaquerizo V, Plasencia MA, Arribas I, et al. Comparison of intra-articular injections of plasma rich in growth factors (PRGF-Endoret) versus Durolane hyaluronic acid in the treatment of patients with symptomatic osteoarthritis: a randomized controlled trial. Arthroscopy 2013;29(10):1635-43.

[42] Pham T, van der Heijde D, Altman RD, et al. OMERACTOARSI initiative: Osteoarthritis Research Society International set of responder criteria for osteoarthritis clinical trials revisited. Osteoarthritis Cartilage 2004;12(5):389-99. 